\title{
Construcción en la identidad profesional en alumnos tutores de un proyecto extensionista ${ }^{11}$
}

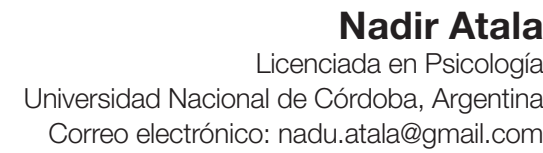

Resumen

El propósito de la investigación fue analizar la construcción de la identidad profesional de alumnos tutores que participaron en un proyecto extensionista durante el año 2018. La relevancia se vincula la necesidad de indagar acerca de los indicadores implicados, para comprender cómo se lleva adelante la construcción de la identidad profesional en adolescentes que ejercen como alumnos tutores. Se llevó a cabo un estudio cualitativo descriptivo, con entrevistas semiestructuradas que se aplicaron en una muestra de 22 alumnos tutores, de ambos sexos. Fueron tomadas de manera individual, pudiendo aprehender de manera más exhaustiva el proceso. Se observó que los jóvenes pudieron recrear las experiencias vividas, darles sentido y apropiarse de la identidad profesional, a partir de las historias que fueron re-construyendo en las entrevistas. Los hallazgos de la presente investigación demuestran la importancia de promover identidades profesionales positivas que resguarden la salud mental de todos los miembros del proyecto y, a su vez, favorezcan el proceso enseñanza-aprendizaje, sin dejar de considerar que a esta práctica compleja se le suma otro proceso, a saber, la adolescencia.

Palabras clave Identidad, adolescentes, entrevista narrativa, autorrelatos.

11 Para citar este artículo: Atala, N. (2022). Construcción en la identidad profesional en alumnos tutores de un proyecto extensionista. Informes Psicológicos, 22(1), pp. 181-200. http://dx.doi.org/10.18566/infpsic.v22n1a11 


\title{
Construction of the professional identity in student tutors of an extension project
}

\begin{abstract}
The purpose of the research was to analyze the construction of the professional identity of student tutors who participated in an extension project during 2018. The relevance is linked to the need to inquire about the indicators involved, in order to understand how the construction of the professional identity of adolescents who act as student tutors is carried out. A descriptive qualitative study was conducted, with semi-structured interviews that were applied in a sample of 22 student tutors, of both sexes. They were taken individually, so as to apprehend the process in a more exhaustive way. It was observed that the young people were able to recreate the experiences they had lived, give them meaning and appropriate their professional identity, based on the stories that were re-constructed in the interviews. The findings of this research demonstrate the importance of promoting positive professional identities that protect the mental health of all the members of the project and favor the teaching-learning process, while still considering that this complex practice is coupled with another process, namely adolescence.
\end{abstract}

\section{Keywords}

Identity, adolescents, narrative interview, self-stories.

\section{Construção da identidade profissional em alunos tutores de um projeto de extensão}

Resumo

0 objetivo da pesquisa foi analisar a construção da identidade profissional de alunos tutores que participaram de um projeto de extensão ao longo de 2018. A relevância está ligada à necessidade de indagar sobre os indicadores envolvidos para compreender como se dá a construção da identidade profissional de adolescentes que atuam como tutores de alunos. Realizou-se um estudo descritivo qualitativo, com entrevistas semiestruturadas, aplicadas em uma amostra de 22 alunos tutores, de ambos os sexos. Eles foram entrevistados individualmente, podendo apreender o processo de forma mais exaustiva. Observou-se que os jovens conseguiram recriar as experiências vividas, dar-lhes sentido e se apropriar de sua identidade profissional, a partir das histórias que foram reconstruindo nas entrevistas. Os achados desta pesquisa demonstram a importância de se promover identidades profissionais positivas que protejam a saúde mental de todos os integrantes do projeto e, por sua vez, favoreçam o processo ensino-aprendizagem, considerando que a essa prática complexa se soma outro processo, a saber, a adolescência. 


\section{ntroducción}

La construcción de la identidad surge de la confrontación de los individuos con preguntas vinculadas a su existencia, tales como: ¿Quién soy?, ¿Quiénes son los otros?, ¿Cuáles son las semejanzas y diferencias? (Lagarde, 1990). Esta situación, según Restrepo (2010), puede considerarse como el punto de partida para la constitución de una identidad como sujetos autónomos.

La identidad personal entonces, parte de la relación entre "la identidad atribuida por otros y la identidad asumida" (Bolívar, Fernández \& Molina, 2005, p. 4), creando una versión identitaria única y singular, en permanente reconfiguración, donde el individuo adopta un carácter activo (Lagarde, 1990).

En estrecha relación dialéctica con la identidad personal, se encuentra la identidad profesional. Entendida como la forma en que los sujetos se definen a sí mismos y son definidos por otros, dentro del entorno profesional e institucional en el que desarrollan su actividad (Bolívar et al., 2005).

En esta investigación se indagó la construcción de la identidad profesional en adolescentes que se desempeñaron como alumnos tutores, dentro de un proyecto extensionista denominado Orientación y Contención de Alumnos tutores. La función de los jóvenes consistió en proporcionar apoyo escolar a escuelas primarias públicas. Esto implicó brindar contención y orientación para que se logren, en la medida de lo posible, aquellos conocimientos y habilidades que permitieran el éxito escolar (Álvarez-Aguilar, Marín-Rodríguez \& Torres-Bugdud, 2012).

Teniendo en cuenta que el alumno tutor comparte algunas similitudes con la tarea docente, se consideró importante indagar el proceso de construcción de la identidad profesional, dado que así se podrán comprender mejor aquellos factores que inciden en el devenir de este rol.

De este modo, el objetivo general de esta investigación se centró en analizar la construcción de la identidad profesional de los alumnos tutores que participaron en un proyecto extensionista Orientación y Contención a Alumnos Tutores durante el año 2018.

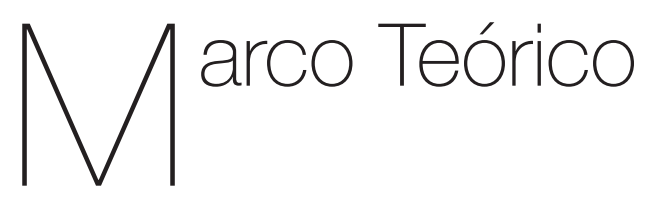

Frente a los conflictos en el sistema educativo surge el alumno tutor (Capelari, 2009), que se convierte en un factor de socialización, integración y facilitador del intercambio multidireccional. Asimismo, responde a las necesidades y problemas de los alumnos a partir de una ayuda personalizada, tanto académica como personal (Álvarez-Aguilar et al., 2012).

Esto le permite desplegar estrategias educativas que apelan a la creatividad, re-significando los espacios educativos y construyendo en conjunto con los niños los espacios de apoyo. El tutor reflexiona, cuestiona e interroga de forma permanente lo que ha adquirido en la práctica y que, ahora, debe explicar (Fernández, 2016). Así, sustituyen la concepción 
tradicional de transmisión de conceptos por la construcción del saber o de saberes (Ariza-Ordóñez \& Ocampo-Villegas, 2004).

\section{La identidad}

La cultura es internalizada por los sujetos, a la vez que subjetivada y apropiada, en un determinado contexto de significaciones compartidas por la comunidad. Por tanto, la identidad es histórica y situada. Se puede decir que es un proceso dinámico, relacional y dialógico que se construye en relación a la otredad, mediante las relaciones sociales (Marcus, 2011).

Es aquel carácter que hace a lo singular, distinto a los demás (Mercado, 2004 en Pérez-Colunga, 2011) y está relacionada con la imagen que se tiene de uno mismo, más que con la realidad de lo que uno es. Esto da lugar a la constitución de la identidad a partir de la demarcación de un yo y otro. Esta relación con otro, con lo que no se es, permite conformar la propia identidad (Marcus, 2011).

\section{La identidad adolescente}

La identidad es "(...) una articulación compleja y multidimensional de elementos psicológicos, sociales, culturales e íntimo-afectivos" (Díaz-Sánchez, 2006, p. 433). Por tanto, la adolescencia va a variar en función de la sociedad y la cultura en la que cada sujeto se desarrolle.

Se puede entender como un período de crisis constitutiva de la identidad, dado que allí se produce una resignificación de los elementos que constituyeron la infancia. Sin embargo, también permite la formación y consolidación de una identidad personal que funcionará como base para las futuras re-significaciones (ZacarésGonzález, Iborra-Cuéllar, Tomás-Miguel \& Serra-Desfilis, 2009).

En resumen, se puede decir que se trata de un período de vulnerabilidad, pero también de cambio y avance físico, intelectual, moral, social, que da como resultado una nueva organización de la identidad (Merino, 1993, citado en Gómez-Palacio \& Carrasco-Tapias, 2010).

\section{La identidad profesional}

Mediante el desarrollo de la práctica de alumno tutor se construye la identidad profesional. En el devenir de la práctica se proyectan, sobre el tutorado, algunos aspectos vividos durante el propio trayecto académico, es decir, que "(...) las experiencias de vida se hacen presentes al momento de ejercer el rol como tutores" (Pérez-Colunga, 2011, p. 4). Así se configura una dualidad que, por un lado, posibilita la continuidad de las vivencias $y$, por otro, representa una ruptura con estas experiencias dando lugar a una re-significación.

La identidad del alumno tutor entonces se conforma a partir de las significaciones sociales que los jóvenes tienen de la actividad que desempeñan (PérezColunga, 2011). A partir de esto se da un proceso de reflexión sobre las creencias y valores propios que confrontan con los sostenidos por la institución educativa y 
determinados por el rol (Conde-Barbosa, Alfonsi, Cipriano \& Placco, 2014).

Desde el Proyecto se generó un espacio para que el grupo de alumnos tutores se pudiera reunir, con el objetivo de que reflexionen, se encuentren, recreen y organicen dentro de un aprendizaje grupal (Jorge, Guzmán \& González, 2016), permitiendo el intercambio de experiencias del grupo de pares.

Los sujetos recrean las experiencias vividas, con el objetivo de darles sentido, a través de autorrelatos. Estos surgen como medio que interrelaciona pasado, presente y futuro (Bolívar et al., 2005) y, además, permiten que los acontecimientos pasados sean resignificados en el presente, dotando a los individuos de herramientas necesarias en el caso de que acontezcan situaciones similares.

El relato de los acontecimientos propios posibilitan apropiarse de una identidad, ya que pueden reconocerse en las historias que cuentan (Cole \& Knowles, 2001, citados en Bolívar et al., 2005); asimismo, Bolívar et al. (2005) sugieren indicadores relacionados con la identidad profesional y con la construcción de la misma, que se hacen presentes en los autorrelatos. Estos son: autoimagen (refiere a cómo se percibe en el desempeño de sus actividades); reconocimiento social (cómo el sujeto describe lo que el entorno institucional y de pares expresa sobre su experiencia como tutor); grado de satisfacción (en qué medida el sujeto considera que esta experiencia de tutoría enriquece su trayectoria tanto profesional como personal); relaciones sociales en la institución (cómo es la relación con el grupo de tutores, los coordinadores, los niños y la escuela a la que asisten estos últimos); actitud ante el cambio (reacciones frente a situaciones inesperadas); competencias (con qué habilidades cuenta para desarrollar el rol y cuáles podría mejorar o adquirir); y expectativas a futuro (qué espera del ejercicio del rol, cuáles son sus metas).

Por otro lado, aquellos indicadores de la construcción de la identidad profesional son: trayectoria de vida (eventos significativos que han llevado a que elija desempeñar el rol); formación (desempeño de actividades en estrecha relación con el ejercicio del alumno tutor); y crisis de identidad (conflictos generados por el desempeño del rol, con respecto a su identidad personal).

\section{М etodología \\ Tipo de estudio y Diseño}

Se llevó a cabo un estudio de tipo cualitativo descriptivo que brindó una perspectiva sobre la variable bajo estudio: la construcción de la identidad profesional (León \& Montero, 2003) y permitió profundizar en la experiencia particular de cada alumno tutor.

El diseño de la investigación se centró en técnicas de recolección de datos centradas en una investigación biográficonarrativa (Bolívar et al., 2005). Teniendo en cuenta que la identidad profesional es una construcción social, deben tenerse en consideración las significaciones y experiencias subjetivas de los adolescentes 
(Bruner, 1991; Sarbin, 1986, citados en Flick, 2004).

Los instrumentos de recolección de datos fueron entrevistas semiestructuradas individuales y observaciones no participantes. Estas últimas permitieron un análisis situado de la muestra en su conjunto (Flick, 2004), para luego comparar con las entrevistas individuales, dando lugar a que los alumnos tutores describieran con palabras propias sus condiciones (Miles \& Huberman, 1994, citados en Sañudo, 2006).

\section{Participantes}

La población objeto de estudio estuvo conformada por alumnos que participaron del proyecto extensionista "Orientación y Contención a Alumnos Tutores" de dos escuelas públicas pre- universitarias de la Ciudad de Córdoba, Argentina.

La muestra fue de tipo no probabilística intencional y se encontró delimitada por el acceso previo en la institución dado el trabajo de prácticas que se desarrollaron.

Los criterios de inclusión implicaron como requisitos: participar del proyecto extensionista durante el año 2018, asistir a los encuentros con el equipo de profesionales a cargo del proyecto y aceptar formar parte de la investigación de manera voluntaria (mediante un consentimiento informado). No se especificaron criterios de exclusión, por ello la muestra no es representativa del total de la población que participa del proyecto extensionista.

La muestra final quedó constituida por 22 alumnos tutores, de ambos sexos, de los cuales 18 fueron de género femenino y 4 de género masculino.

\section{Instrumentos}

Con el objetivo de recolectar datos para la investigación, se utilizaron los siguientes instrumentos: registro en el cuaderno de campo y entrevistas biográficonarrativas individuales.

\section{Registro en el Cuaderno de}

Campo

Éste se llevó a cabo a partir de una observación no participante, tomando en cuenta los diálogos que estuvieran en estrecha vinculación con la construcción de la identidad del tutor.

Se tuvieron en cuenta los siguientes temas: 1) elección del Proyecto (expectativas y temores); 2) acceso al Proyecto (primeros encuentros- impresiones, preocupaciones, planificación de las clases, valoraciones- y desarrollo del rol- momentos críticos al ejercer el rol-); 3) percepción de las propias tareas de enseñanza (quién es un "buen alumno tutor"); y 4) espacios de orientación y contención (otros temas que se deberían trabajar, encuentros más enriquecedores).

\section{Entrevistas Biográfico-} Narrativas Individuales (Bolívar et al., 2005)

La entrevista tiene la función de indagar sobre distintos focos de interés (la trayectoria personal, la identidad del tutor y la vivencia actual acerca del ejercicio del rol). Es una entrevista 
semiestructurada que está dividida en tres partes, conformada por diez ítems y tiene como objetivo un ejercicio reflexivo de autodescubrimiento.

A los fines de este estudio, se llevó a cabo una adaptación de la entrevista original. En primer lugar, dado que la versión original proviene de España, han sido modificados algunos términos utilizados, reemplazándose por aquellos que son de uso corriente en Argentina (vosotros por nosotros, por ejemplo). En segundo lugar, la entrevista original fue tomada en tres tiempos distintos que se corresponden con las partes en que está dividida; sin embargo, por el encuadre en el que se lleva a cabo esta investigación, se hizo necesario reducirla a un solo momento.

\section{Procedimiento y análisis de datos}

La presente investigación se efectuó a partir de diferentes momentos. En un primer momento se realizaron observaciones no participantes, en el marco de los encuentros de capacitación y contención a los que asistieron los tutores.

En un segundo momento, una vez informados los tutores sobre los objetivos de la investigación y solicitado un consentimiento voluntario, fueron convocados a las entrevistas de forma individual. Y, luego, en un tercer momento, se analizaron en relación a los indicadores de identidad profesional, identificando recurrencias y convergencias dentro de las trayectorias de cada tutor.

En un cuarto y último momento, la información se organizó en categorías y temas; se describieron las experiencias de los adolescentes bajo su óptica, su lenguaje y con sus expresiones, para así comprender en profundidad el contexto que rodea a los datos. Luego se interpretaron y evaluaron las categorías y temas, a partir de lo cual se pudieron generar hipótesis y explicaciones de la problemática en estudio.

\section{Consideraciones éticas}

El estudio tuvo como principio fundamental el respeto a las personas y el bienestar de aquellas que decidieron participar voluntariamente. Esto conlleva entender a los sujetos como protagonistas activos de sus decisiones, con capacidad de ser informados de la totalidad del proceso (Miles \& Huberman, 1994, citados en Sañudo, 2006).

En un primer lugar, se les otorgó un consentimiento informado. Éste explicaba los objetivos de la investigación para ser leído y firmado, solo en caso de estar de acuerdo. En segundo lugar, se veló por la confidencialidad y privacidad de los adolescentes, utilizando el material para fines científicos del presente estudio, protegiendo la información personal, evitando la pérdida, adulteración y/o tratamiento no autorizado. Dado que la muestra estaba conformada por jóvenes menores de edad que no pueden dar consentimiento para que su nombre sea utilizado, se decidió aplicar designaciones como alumnos tutores, adolescentes y sujetos, con el objetivo de resguardarlos (Miles \& Huberman, 1994, citados en Sañudo, 2006). 


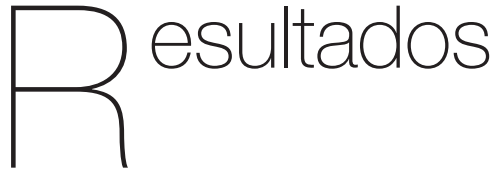

En el presente apartado se presenta la información obtenida a partir de las entrevistas y los registros realizados en las observaciones no participantes.

Caracterización de las experiencias biográficas en torno a la escolaridad y las prácticas de enseñanza en los alumnos tutores

Los adolescentes recordaron su trayectoria por la escuela primaria y brindaron respuestas que pueden agruparse en dos categorías: recuerdo con docentes e influencia de los recuerdos en la elección del proyecto. En los recuerdos con docentes se recuperaron experiencias con docentes que estimulaban cualidades y compartían actividades extra-académicas:

“(...) nunca me olvido cuando estaba

en tercer grado (...) mandaron un suplente (...) Y este profesor dibujaba muy bien, y con la señorita nunca hacíamos nada, entonces a mí me encantaba (...) Y me acuerdo que una vez dijo "acá veo futuros artistas" y me miraba a mí especificamente (...) Y eso fue una de las primeras veces que empecé a dibujar bien" (alumna tutora, 16 años); "(...) en cuarto grado me acuerdo tenía una maestra que tenía una muy buena relación y la había invitado a un concierto que yo tenía (...) me acuerdo que ella fue (...) es como que eso me puso re contenta porque ella se había tomado el tiempo de ir a verme" (alumna tutora, 17 años).

Por otro lado, los entrevistados hicieron mención a la potencial sobre la influencia de sus recuerdos en la elección del proyecto:
"(...) pienso que hay muchos chicos que capaz que los profesores no les dan... perdón por la palabra, no les dan bola (...) Lo digo porque me pasó" (alumno tutor, 17 años); "sí, mucho. Porque al yo haberla pasado tan bien creo que es una forma en la que puedo ayudar al resto" (alumna tutora, 18 años).

Los adolescentes también hicieron mención a las fantasías y temores con respecto al rol. En relación a las fantasías, expresaron deseos vinculados a diversos aspectos. Las más nombradas fueron: aprendizaje de contenidos académicos, por parte del niño; aprender del rol de alumno tutor y formar un vínculo.

Por otro lado, manifestaron algunos de sus temores con respecto al ejercicio del rol, vinculados a la ausencia de capacidades para explicar, de conocimientos y aptitudes para establecer un vínculo. Algunos de los más expresados fueron: no ejercer bien el rol y no poder establecer un buen vínculo con el niño.

Identificación de los aspectos que incidieron en la elección voluntaria para ejercer el rol de alumno tutor

A partir de la participación se construyeron diversas valoraciones, clasificadas 
en dos categorías: emocional y funcional. Lo emocional hace referencia a los sentimientos que surgieron en los jóvenes frente contacto con los niños:

"el afecto que nos podemos dar mutuamente es bastante lindo" (alumna tutora, 17 años); "Para mí es hermoso, no hay otra forma de describirlo" (alumno tutor, 17 años).

La segunda categoría se relaciona con las valoraciones en torno a la utilidad del proyecto. Entre las funcionalidades se puede mencionar la apropiación de una experiencia enriquecedora para su futuro profesional y el desarrollo de herramientas de enseñanza:

"como una actividad personal, algo que hacía en mí tiempo libre" (alumno tutor, 17 años); "creo que es una buena herramienta para mi futuro" (alumna tutora, 16 años); "me ayudó bastante en lo que yo quería hacer de poder salir de ese momento de no saber para qué servía yo" (alumna tutora, 17 años).

\section{Descripción de la autoimagen} de los alumnos tutores

La autoimagen remite a cómo cada alumno tutor se percibe ejerciendo el rol. Las respuestas pueden agruparse en dos categorías: enfoque de la tutoría y habilidades. El enfoque de la tutoría permitió que los adolescentes jerarquizaran entre los aspectos lúdicos, académicos y vinculares, según sus intereses:

"(...) no lo voy a sentar en una silla

y ponerme a hacer ejercicios con él, sino vamos a terminar jugando un juego de mesa (...)" (alumno tutor, 17 años); "Creo que hago más contención" (alumna tutora, 17 años).

Las habilidades remiten a herramientas o estrategias que los adolescentes poseían o fueron adquiriendo durante las tutorías:

\begin{abstract}
"Ahora más al último ya sabía cómo hacer, por ejemplo, para que me traiga las cosas la próxima clase" (alumna tutora, 17 años); "tengo buen trato con los nenes, los entiendo" (alumna tutora, 18 años).
\end{abstract}

Identificación de la valoración y el reconocimiento social que los alumnos tutores perciben de su rol

El ejercicio de los alumnos tutores se da en el marco de dos escuelas primarias. Los jóvenes hicieron referencia a las valoraciones de las instituciones en función de su práctica y las respuestas se agruparon en dos categorías: apoyo institucional e impacto de la valoración institucional. El apoyo institucional hace referencia a cómo los jóvenes interpretan el trato de parte de ambas instituciones:
"nos apoyaron bastante, se nota que querían formar parte del proyecto" (alumna tutora, 17 años); "hay poca participación, por ejemplo, de los profesores en el proyecto" (alumno tutor, 17 años); "Nos daban bastantes libertades a la hora de las observaciones 
que tuvimos al principio" (alumna tutora, 16 años).

La segunda categoría, impacto de la valoración institucional, permitió que los adolescentes expresaran cómo les afectaba el trato de las instituciones:

"Si es una escuela en donde te tratan como si fueras un intruso, sería como que vos vas y estás triste o algo así. No tenes todas las ganas de ir y de ayudar" (alumna tutora, 17 años); "las maestras estaban muy desbordadas, entonces que me parece que valoraron realmente el hecho de que vayamos y estemos con los niños" (alumna tutora, 18 años).

En relación a los momentos que los jóvenes utilizan para compartir experiencias, las respuestas que brindaron pueden agruparse en una categoría: momentos de intercambio. Los entrevistados mencionaron en qué circunstancias se llevaron a cabo los encuentros entre pares:

"nosotros después de hacer las tutorías teníamos reuniones, necesarias y obligatorias" (alumna tutora, 17 años); "hicimos un par de veces la puesta en común" (alumna tutora, 18 años); "cuando volvíamos de las tutorías íbamos contando qué habiamos hecho" (alumna tutora, 17 años).

Las principales fuentes de conflicto que se presentaron al momento de ejercer el rol de alumno tutor, pueden dividirse en dos categorías: conflictos e intentos de solución. Entre los conflictos los jóvenes mencionaron el tiempo de clases, los estados de ánimo, las dificultades para establecer un vínculo, el cambio constante de niños, la distribución del espacio, la falta de coordinación con las maestras, entre otros:
"yo considero que el tiempo era muy poco" (alumna tutora, 17 años); "el hecho de estar trabajando con otros chicos en la misma aula" (alumna tutora, 17 años); "la dispersión. Había tres chicos que eran muy dispersos" (alumna tutora, 17 años).

En la segunda categoría, los alumnos tutores expresaron los intentos que llevaron adelante para solucionar las dificultades; entre ellos, mencionaron el diálogo y la intervención de las maestras:

"No sabíamos cómo manejar, pero para nada la situación. Hasta que llegó la profe y ahí nos ayudó, calmó los nenes" (alumna tutora, 17 años); "no es que los nenes no sean capaces sino como que te das cuenta que lleva su tiempo" (alumna tutora, 17 años); "hicimos lo que pudimos" (alumna tutora, 18 años).

El desarrollo del rol de alumno tutor permitió rescatar las siguientes categorías: competencias emocionales, vínculo niño-alumno tutor y herramientas de enseñanza. Dentro de las competencias emocionales los adolescentes mencionaron la paciencia, empatía, capacidad de escucha, tolerancia a la frustración y la confianza:

"me sentí mejor yo porque la
escuchaba y si era algo que 
necesitaba una respuesta, respondía, y si no, escuchaba" (alumna tutora, 17 años); "un poco de tolerancia a la frustración, por ahíporque uno tiene que como que sobreponerse a esas situaciones" (alumna tutora, 16 años).

En relación al vínculo niño-alumno tutor se hizo referencia a la capacidad de saber que necesita el niño, cómo comunicarse, respetarlos y conocerlos:

"no me creía capaz de hacerlos jugar o de hablar tanto con ellos, porque a mí me cuesta mucho hablar o preguntar cosas o dar consejos. Fue como que eso no me creía tan capaz. Y después vi que sí, podía hacerlo" (alumna tutora, 18 años); "no sé si en la parte académica, pero sí en la parte de saber cómo crear un vínculo con un niño" (alumna tutora, 17 años).

Por último, manifestaron las herramientas de enseñanza que utilizaron:

"yo creo que sí en el pensar actividades para ayudarla. No sé, hacer algún juego para poder ayudar, que sea más dinámico y más fácil de explicar (...)" (alumna tutora, 16 años); "mejorar mío o encontrar otros métodos" (alumna tutora, 17 años).

Caracterización de la formación en el desarrollo de su rol en los alumnos tutores

El equipo de psicólogas a cargo del proyecto de extensión llevó a cabo espacios de encuentro, con el objetivo de que los adolescentes pudieran expresar y trabajar diversas temáticas referidas al rol. Se conformó una categoría: aspectos enriquecedores de los encuentros. Entre ellos mencionaron el acompañamiento constante, las herramientas para construir vínculos y las recomendaciones para afrontar los conflictos:

"También lo del vínculo puede hacer crecer al niño y lo puedo ayudar en otras cosas, que tal vez también le ayude a aprobar (...) Y podemos hacer unas actividades afectivas en el encuentro, no tan solo académicas" (alumna tutora, 17 años); "(..) pude aprender un poco de psicología, del rol de tutor como una relación de hermandad con el alumno, de fraternidad. $Y$, sobre todo, ver cómo muchas veces desarrolla apegamiento o cariño con el niño (...)" (alumno tutor, 17 años).

Los jóvenes respondieron sobre sus sentimientos respecto al rol y a partir de ello se creó una categoría: tipo de sentimientos. En este sentido, manifestaron qué les provocó ser tutor de niños:

"me siento muy feliz (...) es uno de los plus más importantes para mí y durante el año de las cosas más lindas que hice" (alumno tutor, 17 años); "me sentía re mal, porque a pesar de que era muy poco el tiempo que vamos, cada una semana; y a veces no íbamos, entonces era como una pérdida de tiempo. Sentía que podía haber hecho más" (alumna tutora, 17 años). 


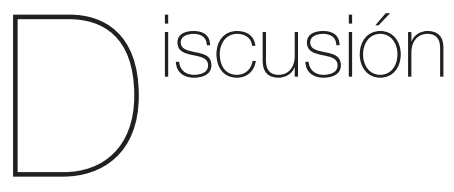

Experiencias biográficas en torno a la escolaridad y las prácticas de enseñanza en los alumnos tutores

La muestra puso en evidencia que los alumnos tutores eligieron formar parte del proyecto a partir del recuerdo de sus experiencias, pudiendo retomar de las figuras docentes las metodologías utilizadas. Entonces los recuerdos no solo fueron utilizados como referencias para participar, sino también que se conforman como bases desde las que se vinculan, imaginariamente, con los niños a quienes van a tutoriar.

Por otro lado, las experiencias pasadas posibilitan la construcción de una identidad basada en continuidades y rupturas (Pérez-Colunga, 2011), retomando aspectos positivos y negativos de sus trayectos, con el fin de reproducirlos o revertirlos. Es así que opuestos y complementarios van enriqueciendo la propia identidad y la práctica misma. Siguiendo a Alliaud (2004), se puede decir que la escuela recorrida se conforma como un espacio al que se regresa, como docente, para enseñar, siendo aquellas experiencias pasadas las que proporcionan el sustento a las prácticas actuales.

En este sentido, los jóvenes enfatizan la importancia del afecto, el sostén, la escucha y el compromiso con la enseñanza como aspectos fundamentales en los docentes de sus escuelas primarias, siendo, estos trayectos, marcas que constituyen las historias subjetivas (Jorge, Guzmán, Muñoz \& García, 2018a).

El ejercicio implica la construcción de una identidad y posibilita la vivencia personal de un rol reservado a los docentes. Según Pérez-Colunga (2011), este proyecto genera una ruptura de las vivencias, dando lugar a una re-significación de las tareas y el rol docente. Los adolescentes brindan ayuda personalizada académica y emocional (Álvarez-Aguilar et al., 2012), preocupándose por el aprendizaje y por proveer enseñanzas de vida, ayuda y contención ante problemas que exceden el ámbito educativo. De esta manera, se entienden como mediadores entre el niño y las realidades sociales y se posicionan como agentes de cambio social (ArizaOrdóñez \& Ocampo-Villegas, 2004).

Las motivaciones se encuentran vinculadas, por un lado, a los intereses de los jóvenes y, por otro, a las fantasías y temores en torno al rol, de modo tal que las expectativas aparecen en función del apoyo y la orientación para el logro de conocimientos, capacidades y habilidades que permitan alcanzar óptimos rendimientos escolares (Álvarez-Aguilar et al., 2012); al mismo tiempo que se relacionan con los imaginarios acerca del ejercicio docente, a partir de los cuales establecen las metas que se proponen alcanzar. En este sentido, las fantasías resultan datos útiles dado que posibilita observar el recorte de la profesión que cada sujeto realiza (MillerFlores, 2002, citado en Jorge et al., 2018b).

Por otro lado, los temores de los alumnos tutores remiten a capacidades, conocimientos y habilidades consideradas como fundamentales en la práctica, como también a las posibilidades 
de establecer vínculos amorosos y comprometidos.

Las fantasías y temores surgen en el devenir de las trayectorias personales, por lo tanto, la elección no puede asociarse a condiciones innatas. Por ello, ni la profesión ni la identidad pueden ser entendidas fuera del contexto psico-socio-educativo en el que se producen.

Aspectos que incidieron en la elección voluntaria para ejercer el rol de alumno tutor

Las tutorías se conciben como espacios nutricios a nivel emocional y experiencial, posibilitando en los jóvenes una redefinición de su identidad (Díaz-Sanchez, 2006). Las valoraciones en relación a la experiencia tuvieron múltiples anclajes: la aprehensión de las distintas condiciones socio-económicas, para buscar una participación activa que propicie la transformación social, y la significación como ámbitos de sostén y afirmación identitaria. A partir de esto los alumnos tutores exploran sus aspiraciones profesionales, utilizando el espacio como ensayo de lo que sería su futura profesión docente; sin embargo, también le dan sentido a su existencia, haciendo las veces de un factor ordenador de la vida.

El proyecto extensionista es una alternativa para explorar las distintas visiones de mundo (Tesouro-Cid et al., 2013), conocer realidades ajenas y brindar sentido y dirección a sus respectivos proyectos de vida.

El primer encuentro de los jóvenes con los niños se da a partir de observaciones no participantes en el aula y jornadas lúdicas. Estos espacios permiten un acercamiento entre los sujetos que conformarán, en una instancia próxima, la díada (niño-alumno tutor), sentando las bases para la conformación del vínculo. Y, por otro lado, los jóvenes refutan o confirman los temores y fantasías (desarrollados en el apartado anterior).

El docente está sometido a presiones, carencias y conflictos que son manifestados a nivel psicológico (Iriarte-Díaz-Granados, 2010) y representan un obstáculo en el proceso enseñanza-aprendizaje. Estos sentimientos resurgen en los adolescentes cuando se presentan conflictos, afectando la construcción de una identidad positiva, tanto profesional como personal, que contemple las dificultades como desafíos y no como estancamientos emocionales. No obstante, frente a las adversidades del ejercicio, han buscado diversas soluciones utilizando sus propias herramientas o la ayuda del equipo de Orientación y Contención. Esto pone de manifiesto la necesidad de sostener un espacio en donde se sientan contenidos, escuchados y comprendidos, teniendo en cuenta que la adolescencia como etapa evolutiva implica un período de vulnerabilidad creciente (Gómez-Palacio \& Carrasco-Tapias, 2010).

\section{La autoimagen de los alumnos tutores}

La autoimagen o autopercepción puede ser entendida como la concepción que el sujeto construye de sí, en relación al ejercicio del rol del alumno tutor.

El ejercicio del rol los ubica frente a las tareas docentes de diversos modos y permite una apreciación de sí mismos. 
Esto va conformando la autoimagen. Los jóvenes que se perciben incapaces de llevar adelante la práctica manifiestan sentimientos de frustración e incapacidad para afrontar dificultades, poniendo de relieve las limitaciones como aspectos imposibles de modificar. Generan así una percepción de sí negativa que impacta directamente en la construcción de la propia identidad profesional y, a su vez, en el vínculo con el niño, dando como resultado sentimientos de frustración e insatisfacción (Mares-Miramontes et al., 2009).

No obstante, aquellos alumnos tutores que reconocen los límites y conflictos como parte constitutiva del rol, reinventan su práctica de forma permanente para adaptarse a las necesidades. Esto conlleva la construcción de una imagen positiva de sí mismos, comprendiendo los conflictos como oportunidades para el desarrollo de herramientas que enriquezcan el rol. Esta autopercepción da la posibilidad de identificarse como parte de un todo, de un proyecto que los respalda y contiene y, a la vez, brinda seguridad y confianza en los propios conocimientos.

\section{Valoración y el reconocimiento} social que los alumnos tutores perciben de su rol

A partir de la valoración y el reconocimiento que hacen las instituciones en relación al rol, los alumnos tutores reconocen la relevancia de su tarea. La construcción de la identidad profesional y la autoimagen dependen, también, de estos factores (Bolívar, 2004, citado en Alliaud \& Vezub, 2012).
En este sentido, las valoraciones impactan en el ejercicio. Por tanto, el apoyo que los jóvenes reciben por parte de la institución es significado como una valoración positiva que brinda sentimientos de comodidad y seguridad. Este reconocimiento es fundamental debido a que da equilibrio y promueve el desarrollo personal al momento de las prácticas (Sebastián, 2012).

No sorprende que el reconocimiento primordial, para los jóvenes, sea el vínculo con los niños, ya que el aprendizaje depende de la modalidad vincular establecida, siendo un obstáculo o facilitador para el acceso al conocimiento (Torcomian, 2006).

Sin embargo, la autoimagen no es el único aspecto que contribuye a la construcción de la identidad profesional, sino que los otros también proveen identificaciones sobre el rol que ejercen. Es decir, que se complementan lo que es atribuido por otros, lo que se espera del rol, y lo que cada adolescente asume como propio.

El grupo de alumnos tutores se conforma como proveedor de herramientas y estrategias; esto hace necesario generar espacios de encuentro que sirvan para intercambiar conocimientos y experiencias que enriquezcan el ejercicio (Jorge et al., 2016). Además, la grupalidad sirve a los fines de sostén y proporciona seguridad en la tarea que llevan a cabo, a la vez que permiten construir una identidad social. Ésta se conforma de dos fuentes: las representaciones sociales que los jóvenes tienen del rol (fantasías, temores e ideales) y la práctica real del rol (Mercado, 2004, citado en Pérez-Colunga, 2011). 
Las valoraciones, reconocimientos y la constitución de la autoimagen determinan las formas como se resuelven los conflictos, enriqueciendo y ampliando las posibilidades del rol cuando los adolescentes buscan soluciones en sus compañeros, equipo de psicólogas o en las coordinadoras del proyecto.

Por otro lado, la introspección de los alumnos tutores permitió sostener el compromiso y la responsabilidad para beneficio de los niños (Voli, 1999, citado en Sebastián, 2012). Esto llevó a que los conflictos dieran cuenta de las limitaciones, virtudes, cualidades y de la idoneidad para enfrentar diferentes situaciones.

Las prácticas del proyecto son heterogéneas e históricas, dependiendo de las propias vivencias y contextos en los que se desarrollan. Dilucidar estas dimensiones les permitió re-significar su ejercicio (Conde-Barbosa et al., 2014), contraponiendo ideales imaginarios y el ejercicio real.

Estos imaginarios conforman, entre otras cosas, el ideal del buen alumno. Es construido antes del contacto con los niños, a partir de las representaciones sociales que sostienen en relación a la práctica y forma parte de la identidad social que es atribuida por los otros. Este ideal promueve la internalización del rol, el cómo deberían ser (Pérez-Colunga, 2011) y conforman el estereotipo del tutor.

Entonces la percepción de crecimiento del rol, una autoimagen positiva, la capacidad de buscar soluciones y las metas cumplidas, son factores que contribuyen al deseo de realizar prácticas futuras similares al proyecto extensionista $\mathrm{o}$, incluso, de repetir la práctica.

\section{La formación en el desarrollo de su rol en los alumnos tutores}

Los espacios de formación son momentos de reflexión, encuentro, recreación y organización en torno a su ejercicio como alumnos tutores (Jorge et al., 2016). A su vez, se aprenden contenidos y formas del ser docente, a partir de la solidaridad del grupo. Por tanto, estos encuentros sirven a los fines de rescatar herramientas, de constituir identidad y brindar conocimientos fundamentales de la tarea (Ávalos, 2005).

Asimismo, el equipo psicológico brinda soporte y contención, permitiendo configurar una autoimagen positiva y fortalecer la identidad profesional de los alumnos tutores. Así se registran sentimientos de sostén que permiten desarrollar habilidades, tomar conciencia de sí y manejar emociones y motivaciones enmarcadas en la práctica (Pegalajar-Palomino \& López-Hernáez, 2015).

La formación docente comienza a partir del primer contacto con los tutorados (Ávalos, 2005), generando inseguridades y conflictos en los jóvenes. Es por ello que se vuelve imprescindible generar conceptos positivos y confianza en sí mismos, con el objetivo de adaptarse a las demandas del proyecto en general y de los niños en particular (Cabanach, Valle, Rodríguez, Piñeiro \& González, 2010, citados en PegalajarPalomino \& López-Hernáez, 2015). 


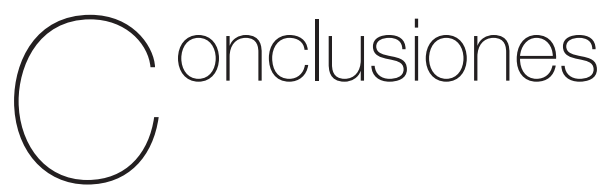

La identidad profesional de los alumnos tutores se constituye en un proceso dialéctico entre lo individual y lo colectivo (Prieto-Parra, 2004) y deviene del ejercicio del rol de alumno tutor.

Según Conde-Barbosa et al. (2014) las idealizaciones de la tarea, de la conducta del niño, de las situaciones que pudieran presentarse y del tratamiento institucional, se contraponen a la realidad. Siguiendo a Prieto-Parra (2011) la identidad profesional puede entenderse como una configuración compleja que se encuentra en permanente (re) construcción, desplegándose en las relaciones sociales que se dan entre los alumnos tutores, los niños, coordinadores y el equipo psicológico. Son estas identificaciones que los otros formulan sobre los alumnos tutores las que construyen las identidades, por lo tanto, el carácter intersubjetivo y relacional es constitutivo (Sayago-Quintana, Chacón-Corzo \& Rojas de Rojas, 2008). Lo que es atribuido como parte del rol, el papel que se les reconoce y lo que cada adolescente registra de sí mismo son las dimensiones que hacen a la identidad profesional (Bolívar et al., 2005).

Este proyecto surge a partir del intercambio entre jóvenes y niños de diferentes estratos sociales, produciendo un choque de costumbres y posibilidades que difieren unas de otras. Esto delimita la particularidad, al estar situada en contexto social determinado y determinada por relaciones sociales específicas (Bolívar et al., 2005).
Las entrevistas brindaron un espacio para apropiarse de la identidad construida durante los meses que duró la práctica, ya que las preguntas que fueron respondiendo permitieron relacionar cómo comenzaron y cuáles habilidades y capacidades aprehendieron en el devenir de la práctica.

La autoimagen y la gestión emocional se destacan como factores determinantes para el equilibrio psicológico, así como para el rendimiento profesional y el desenvolvimiento de relaciones con los demás. Tener en consideración los aspectos desarrollados permitirá llevar adelante tutorías de calidad para todas las personas involucradas en el proyecto. Allí entonces es donde radica la importancia de promover la construcción de identidades profesionales positivas.

En este sentido, la presente investigación contribuye a la comprensión acerca de la identidad profesional de los alumnos tutores, permitiendo reflexionar acerca de qué aspectos deben tenerse en cuenta para un correcto acompañamiento a quienes se encuentran atravesando por las tutorías. Además, pone en evidencia la necesidad de conformar y sostener espacios de contención que tengan en consideración los aspectos constitutivos de la identidad profesional, así como aquellos factores obstaculizadores y conflictivos que pueden afectar su desarrollo positivo.

Por otro lado, la metodología utilizada permitió una mayor comprensión del fenómeno. Las entrevistas individuales posibilitaron a los alumnos tutores que, a la vez que relataban su experiencia, fueran reconociéndose en el recorrido. Además se pusieron en palabras y se nombraron aquellas emociones y sentimientos que 
surgieron en la práctica, pudiendo trabajar sobre ellas, dando un segundo espacio de contención, aparte de los encuentros con el equipo de psicólogas, donde fueron escuchados y, más importante aún, pudieron escucharse.

En relación a las limitaciones de la investigación se pueden mencionar las más influyentes a la hora de llevarse a cabo. En primer lugar, el tipo de muestra, ya que abarcó a todos los jóvenes que participaban del proyecto; sin embargo, el acceso se vio dificultado por el cronograma académico de las escuelas, tanto primarias como secundarias. A su vez, esto imposibilitó indagar las implicaciones del género o la edad, por ejemplo. Por otro lado, el desarrollo de un estudio longitudinal resultó inasequible debido a la transversalidad de la investigación que duró solo un año. Y, en última instancia, el proyecto extensionista impidió un abordaje intersectorial que implicara a todos los miembros que participan (alumnos tutores, niños, coordinadores, maestras, directivos) con el objetivo de un abordaje integral.

No obstante estas limitaciones, los resultados obtenidos a partir de la investigación aportan al enriquecimiento del proyecto de tutorías en general y de los espacios de encuentro, con el equipo de Orientación y Contención a Alumnos Tutores, en particular. Este estudio permite reflexionar sobre la importancia de tomar en cuenta aquellos indicadores que conforman la identidad profesional de los alumnos tutores.

Por último, se puede decir que el proyecto se inscribe en un proceso relacional situado, constituido por diversas crisis (Bolívar, 2006; Bolívar, Domingo \& Pérez, 2014, citados en Jarauta-Borrasca
\& Pérez-Cabrera, 2017). Por eso se hace imprescindible promover identidades positivas que resguarden la salud mental de todos los miembros del proyecto y, a su vez, favorezcan el proceso enseñanzaaprendizaje, sin dejar de considerar que a esta práctica compleja se le suma otro proceso, a saber, la adolescencia, con otro nivel de complejidad.

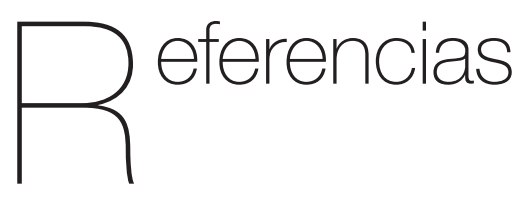

Alliaud, A. (2004). La biografía escolar en el desempeño profesional de docentes noveles. (Tesis de Doctorado). Universidad Nacional de Buenos Aires, Argentina. Recuperado de http://repositorio. filo.uba.ar:8080/bitstream/handle/ filodigital/4100/uba_ffyl_t_2003_49848_ v1.pdf?sequence $=1$ \&isAllowed=y

Alliaud, A. (2004). La experiencia escolar de maestros inexpertos. Biografías, trayectorias y práctica profesional. Revista Iberoamericana de Educación, 34(1), 1-11. https://doi.org/10.35362/rie3412888

Alliaud, A. \& Vezub, L. (2012). El oficio de enseñar: sobre el quehacer, el saber y el sentir de los docentes argentinos. Revista Diálogo Educacional, 12(37), 927952. http://dx.doi.org/10.7213/dialogo. educ.7211

Álvarez-Aguilar, N., Marín-Rodríguez, C. \& Torres-Bugdud, A. (2012). La Interacción Tutor- Estudiante en la Educación Superior: Un Acercamiento a su Diagnóstico. Humanidades Médicas, 12(3), 409-426. Recuperado de http://scielo.sld.cu/scielo. php?script=sci_arttext\&pid=S1727- 
$81202012000300004 \& \operatorname{lng}=$ es\&tIng $=$ es.

Ariza-Ordóñez, G. \& Ocampo-Villegas, H. (2004). El Acompañamiento Tutorial Como Estrategia de la Formación Personal y Profesional: un Estudio Basado en la Experiencia en una Institución de Educación Superior. Universitas Psychologica, 4(1), 31-42. Recuperado de http://www.scielo.org.co/scielo. php?script=sci_arttext\&pid=S1657$92672005000100005 \& \operatorname{lng}=$ en\&tIng $=$ es.

Ávalos, B. (2005). Las instituciones formadoras de docentes y las claves para formar buenos docentes [Archivo PDF]. Recuperado de https://sites.google. com/site/gestiondocentedigital/home/ Clavesparaformarbuenosdocentes.pdf

Ávalos, B. \& Sotomayor, C. (2012). Cómo ven su identidad los docentes chilenos. Perspectiva Educacional, formación de profesores, 51(1), 57-86. Recuperado de https://www.redalyc.org/ pdf/3333/333328167005.pdf

Bolívar, A., Fernández, M. \& Molina, E. (2005). Investigar la identidad profesional del profesorado: Una triangulación secuencial [Archivo PDF]. Recuperado de http://www. qualitative-research.net/fqs-texte/1-05/051-12-s.htm

Capelari, M. (2009). Las Configuraciones del Rol del Tutor en la Universidad Argentina: Aportes Para Reflexionar Acerca de los Significados que se Construyen Sobre el Fracaso Educativo en la Educación Superior [Archivo PDF]. Recuperado de https://rieoei.org/historico/ deloslectores/3110Capelari.pdf
Conde-Barbosa, R., Alfonsi, S., Cipriano, E. \& Placco, V. (2014). Movimientos Identitarios del Profesor Principiante. Simposio Internacional: Aprender a Ser Docente en un Mundo en Cambio, Barcelona, España. Recuperado de http://hdl.handle. net/2445/50680.

Díaz-Sánchez, J. (2006). Identidad, Adolescencia y Cultura: Jóvenes Secundarios en un Contexto Regional. Revista Mexicana de Investigación Educativa, 11(29), 431 457. Recuperado de http://www. scielo.org. $\mathrm{mx} /$ scielo. php?pid=S140566662006000200431\&script=sci_arttext

Fernández, C. (2016). Tutorías entre Pares en la Facultad de Psicología: Aportes al Estudiante Tutor en sus Procesos de Enseñanza-Aprendizaje. (Tesis de Doctorado). Universidad de la República Uruguay. Recuperado de https://www.colibri.udelar.edu.uy/jspui/ handle/123456789/8474.

Flick, Y. (2004). Introducción a la investigación cualitativa. Madrid: Ed. Morata SL.

Gómez-Palacio, G. D., \& CarrascoTapias, N. (2010). Construcción de la identidad adolescente en los contextos sociohistóricos y culturales contemporáneos. Pensando Psicología, 6(11), 95-101. Recuperado de https:// revistas.ucc.edu.co/index.php/pe/article/ view/370

Iriarte-Díaz-Granados, F. (2010). La salud mental del docente como mediación del proceso de enseñanza-aprendizaje. Psicología desde el Caribe, (2-3), 24-38. Recuperado de http://rcientificas.uninorte. edu.co/index.php/psicologia/article/ viewFile/677/9461 
Jarauta-Borrasca, B. \& Pérez-Cabrera, M J. (2017). La construcción de la identidad profesional del maestro de primaria durante su formación inicial. El caso de la Universidad de Barcelona. Revista de Curriculum y Formación del Profesorado, 21(1), 103-122. Recuperado de http://hdl. handle.net/2445/122650

Jorge, E., Guzmán, M., Gentes, G., \& González, C. (2016). La práctica extensionista del docente universitario. Reconfiguración del rol en la construcción de la demanda. [Archivo PDF]. Recuperado de http:// beu.extension.unicen.edu.ar/xmlui/ bitstream/handle/123456789/83/La\%20 pr\%C3\%A1ctica\%20extensionista\%20 del\%20docente\%20universitario.\%20 \%20Reconfiguraci\%C3\%B3n\%20 d e l $\% 2$ orol $\% 20$ e n $\% 20$ l a $\% 20$ construcci\%C3\%B3n\%20de\%20la\%20 demanda.pdf?sequence $=1$

Jorge, E., Guzmán, M., Muñoz, L., \& García, A. (2018a). Historias recuperadas, historias por escribir-reflexiones para la construcción del rol de alumno tutor. Revista Brasileira de Extensão Universitária, 9(2), 99-106. https://doi. org/10.24317/2358-0399.2018v9i2.7586

Jorge, E., Guzmán, M., Muñoz, L., \& García, A. (2018b). Percepción de creencias, fantasías y temores de los alumnos tutores y las alumnas tutoras en momentos previos al ejercicio de su práctica. Universidad en Diálogo: Revista de Extensión, 8(1), 23-37. https://doi.org/10.15359/udre.8-1.2

Marcus, J. (2011). Apuntes Sobre el Concepto de Identidad. INTERSTICIOS: Revista Sociológica de Pensamiento Crítico, 5(1), 107-114. Recuperado de http://www. intersticios.es/article/view/6330
Mares-Miramontes, A., Martínez-Llamas, R., \& Rojo-Sabaleta, H. (2009). Concepto y expectativas del docente respecto de sus alumnos considerados con necesidades educativas especiales. Revista Mexicana de Investigación Educativa, 14(42), 969996. Recuperado de http://www.scielo. org.mx/scielo.php?script=sci_arttext\&pid $=$ S1405-66662009000300016

Lagarde, M. (1990). Identidad Femenina. [Archivo PDF]. Recuperado de https:// xenero.webs.uvigo.es/profesorado/ purificacion_mayobre/identidad.pdf

León, O. G. \& Montero, I. (2003). Métodos de investigación en Psicología y Educación ( $3^{a}$ ed.). Madrid: McGraw-Hill

Palomino, M. \& Hernáez, L. (2015). Competencias emocionales en el proceso de formación del docente de Educación Infantil. REICE. Revista Iberoamericana sobre Calidad, Eficacia y Cambio en Educación, 13(3), 95-106. Recuperado de https://www.redalyc.org/ pdf/551/55141402006.pdf

Pérez-Colunga, B. (2011). La Construcción de la Identidad del Tutor de Acompañamiento: Los tutores de la UAM-XOCHIMILCO. XI Congreso Nacional de Investigación Educativa, México. Recuperado de http://www.comie.org.mx/congreso/ memoriaelectronica/v11/docs/ area_16/0938.pdf

Prieto-Parra, M. (2004). La Construcción de la Identidad Profesional del Docente: Un Desafío Permanente, Revista Enfoques Educacionales, 6(1), 29-49. Recuperado de https://revistas.uchile.cl/index.php/REE/ article/view/48128 
Restrepo, A. (2010). Los Jóvenes y sus Luchas por el Reconocimiento. Nómadas, (32), 179-193. Recuperado de http://hdl.handle. net/10495/3729

Sebastián, V. H. (2012). Autoestima y autoconcepto docente. Phainomenon, 11(1), 23-34. https://doi.org/10.33539/phai. v1111.226

Sañudo, L. E. (2006). La ética en la investigación educativa. Hallazgos, 3(6), 83-98. Recuperado de https://www.redalyc.org/ pdf/4138/413835165006.pdf

Sayago-Quintana, Z., Chacón-Corzo, M. \& Rojas de Rojas, M. (2008). Construcción de la Identidad Profesional Docente en Estudiantes Universitarios. Educere: Investigación Arbitrada, (42), 551-561. Recuperado de https://www.redalyc.org/ pdf/356/35614569016.pdf

Tesouro-Cid, M., Palomanes-Espadalé, M., Bonachera-Carreras, F. \& MartínezFernández, L. (2013). Estudios Sobre el Desarrollo de la Identidad en la Adolescencia. Tendencias Pedagógicas, (21), 211-224. Recuperado de http:// www.tendenciaspedagogicas.com/ Articulos/2013 21_15.pdf

Torcomian, C. (2006). Una mirada psicoanalítica de la relación docente alumno en El Banquete de Platón y su proyección en la escuela de nuestro tiempo. Capítulo del libro "Estudios Platónicos II". Ediciones del copista. Recuperado de https://www. aacademica.org/000-054/472

Zacarés-González, J., Iborra-Cuéllar, A., Tomás-Miguel, J. \& Serra-Desfilis, E. (2009). El Desarrollo de la Identidad en la Adolescencia y Adultez Emergente: Una Comparación de la Identidad Global Frente a la Identidad en Dominios Específicos. Anales de Psicología, 25(2), 316-329. Recuperado de https://revistas.um.es/ analesps/article/view/87931/84641 